Chapter 39.

\title{
Sweet Corn Production in Florida
}

\section{BOTANY}

\section{Nomenclature}

Family - Poaceae (Gramineae)

Sweet corn - Zea mays var. rugosa

\section{Origin}

Like bean, potato, tomato, pepper, and pumpkin, sweet corn is of New World origin (Fig. 39-1). However, wild corns have not been found. It is believed that corn which evolved from ancestral types in South America was later domesticated in Central America.

\section{Related Species}

Sweet and popcorn are the only members of the Poaceae family which are classed as vegetables. Nonetheless, this family is one of the most important in providing human sustenance, either directly or as feed for domestic animals, with grains such as wheat, rice, oats, rye, and corn or indirectly as hay and forage grasses for domestic animals. Sugar cane is also an important member of this family.

\section{Pollination}

Sweet corn is wind pollinated, i.e. wind is the agent responsible for transfer of pollen from the tassel to the silk to effect the pollination process. Isolation of genetic types, just as isolation of yellow and white corns of a single genetic type, must occur for them to produce their desired characteristics. Isolation strategies include:

Distance - about 300 feet should be sufficient isolation to avoid cross pollination.

Time - Maturity differences of at least 14 days which can be accomplished by variety selection or successive plantings should be sufficient to avoid cross pollination of genetic types.

\section{SEEDING AND PLANTING}

Planting dates and seeding information are given in Table 3.

\section{VARIETIES}

A description of major sweet corn types currently available is given in Table 1. Supersweet (shrunken-2) sweet corn varieties that are in commercial use in Florida are given in Table 2.

Table 1. Description of major sweet corn types currently available.

\begin{tabular}{|c|c|c|c|}
\hline Type & Genes controlling sweetness & Storage life ${ }^{1}$ (days) & Relative sugar content \\
\hline Sugary & Full complement of sugary & 1 to 3 & Normal \\
\hline $\begin{array}{l}\text { Sugary Enhanced, also } \\
\text { "Modified" and "EH" }\end{array}$ & $\begin{array}{l}\text { Full complement of sugary (su) and half or } \\
\text { full complement of sugary enhancer (se) }\end{array}$ & 3 to 5 & $\begin{array}{l}\text { Slight to moderate } \\
\text { or even high levels }\end{array}$ \\
\hline $\begin{array}{l}\text { Supersweet, also "Ultra," } \\
\text { "Extra" sweet }\end{array}$ & $\begin{array}{l}\text { No sugary (su); full complement } \\
\text { of shrunken-2 (sh } 2)\end{array}$ & 5 to 10 & High \\
\hline $\begin{array}{l}\text { Synergistic, also } \\
\text { "Sweet-Gene Hybrid" }\end{array}$ & $\begin{array}{l}\text { Full complement of sugary (su); and } \\
\left.\text { half complement of shrunken-2 (sh }{ }_{2}\right)\end{array}$ & 3 to 5 & Moderate \\
\hline Improved Supersweet & $\begin{array}{l}\text { Half complement of sugary (su); and } \\
\text { full complement of shrunken-2 }\left(\mathrm{sh}_{2}\right)\end{array}$ & 5 to 10 & Very high \\
\hline ADX & $\begin{array}{l}\text { No sugary (su) or shrunken-2 }\left(\mathrm{sh}_{2}\right) \text { genes; } \\
\text { full complement of ae, du, and wx genes. }\end{array}$ & 5 to 10 & Moderate to high \\
\hline
\end{tabular}




\section{FERTILIZER AND LIME}

For mineral soils with subsurface or sprinkler irrigation, band all $\mathrm{P}_{2} \mathrm{O}_{5}$ and micronutrients with 20 to $25 \%$ of $\mathrm{N}$ and $\mathrm{K}_{2} \mathrm{O}$ at planting. Sidedress band remaining $\mathrm{N}$ and $\mathrm{K}_{2} \mathrm{O}$ in one or two applications during the early part of growth cycle. After midseason, $\mathrm{N}$ and $\mathrm{K}_{2} \mathrm{O}$ can be applied through center-pivot irrigation system at rates of 10 to $20 \mathrm{lbs} / \mathrm{A}$ of $\mathrm{N}$ and $\mathrm{K}_{2} \mathrm{O}$ in several applications.

For mulched crops with subsurface irrigation, broadcast all $\mathrm{P}_{2} \mathrm{O}_{5}$, micronutrients and 20 to $25 \% \mathrm{~N}$ and $\mathrm{K}_{2} \mathrm{O}$ in bed prior to mulching. For subsurface irrigated crops, band remaining $\mathrm{N}$ and $\mathrm{K}_{2} \mathrm{O}$ in groove 2 to 3 inches deep in bed center. For sprinkler irrigation, broadcast all fertilizer in bed.

For organic soils, band all $\mathrm{P}_{2} \mathrm{O}_{5}$ and micronutrients at planting. Broadcast all $\mathrm{K}_{2} \mathrm{O}$. Supplemental $\mathrm{N}$ at rate of $40 \mathrm{lbs} / \mathrm{A}$ might be needed in cool winter weather or after leaching rain.

Soil test and fertilizer recommendations for mineral soils are given in Table 4. Soil test and fertilizer recommendations for Histosols are given in Table 5.

\section{PLANT TISSUE ANALYSIS}

Plant tissue analysis information for sweet corn is given in Table 6. The analysis was done when the plants were 30 inches tall, using the most recently matured leaf.

\section{IRRIGATION}

Water requirements of sweet corn (see Chapter 8, Principles and Practices for Irrigation Management of Vegetables, Table 4 to 6) increase rapidly from about $40 \%$ of ETo during early growth to $110 \%$ of ETo at peak growth (see Chapter 8, Principles and Practices for Irrigation Management of Vegetables, Table 3). Proper water management is essential during the silking and tasseling and the ear development periods. Water requirements may decrease to $100 \%$ of ETo during the final week or two of growth.

\section{WEED MANAGEMENT}

Herbicides labeled for weed control in sweet corn are listed in Table 4.

\section{DISEASE MANAGEMENT}

Chemicals approved for disease management in sweet corn are listed in Table 8.

\section{INSECT MANAGEMENT}

Table 9 outlines the insecticides approved for use on insects attacking sweet corn.

\section{PRODUCTION COSTS}

Average breakeven production costs for sweet corn vary among Florida's production areas. The costs for a given year, 1998 - 99, are outlined for Dade County in Table 10 and Palm Beach County in Table 11.

Table 3. Seeding and planting information for sweet corn.

\begin{tabular}{|lc||}
\hline \multicolumn{2}{|c|}{ Planting dates } \\
\hline \hline North Florida & Feb - Apr \\
Central Florida & Jan - Apr \\
South Florida & Oct - Mar \\
\multicolumn{2}{|c|}{ Seeding information } \\
\hline \hline \multicolumn{2}{|c|}{ Distance between rows (in) } \\
Distance between plants (in) & $28-32$ \\
Seeding depth (in) & $6-8^{1}$ \\
Seed per acre (Ib) & $1.0-1.5$ \\
Days to maturity from seed & $6-15$ \\
Plant population (acre) & $64-90$ \\
\hline${ }^{1}$ Wider rows and between plant spacings will yield larger ears. \\
\hline
\end{tabular}

Table 2. Some supersweet (shrunken-2) sweet corn varieties that are grown in Florida arranged by kernel color.

\begin{tabular}{||lll|}
\hline Yellow & White & Bicolor \\
\hline \hline Beyond Multisweet & Boreal & Big Time \\
Prime Time & Summer Sweet 6801 & Beyond BC \\
Prime Plus & Summer Sweet 7111 & Obsesssion \\
Summer Sweet 6800R & Summer Sweet 7311 & Summer Sweet 6802 \\
Summer Sweet 7100R & Vail & Summer Sweet 7102 \\
Summer Sweet 7650R & & Summer Sweet 8102R \\
Summer Sweet 8100R & & Tethys \\
Winstar & & \\
\hline
\end{tabular}


Table 4. Soil test and fertilizer recommendations for mineral soils for sweet corn. ${ }^{1}$

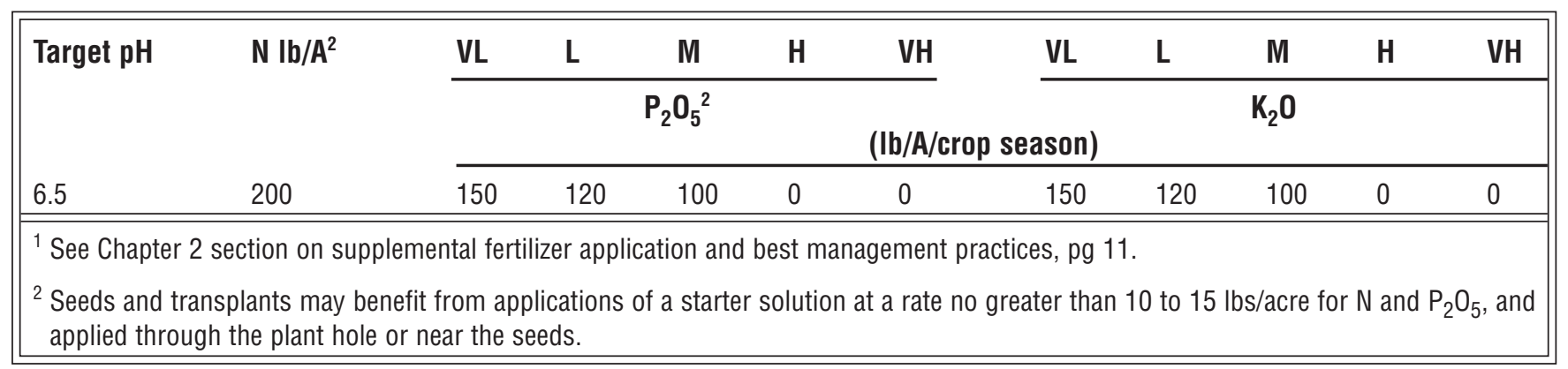

Table 5. Soil test and fertilizer recommendations for Histosol soils for sweet corn, with target $\mathrm{pH}=6.5$ and $\mathrm{N}$ rate $0 \mathrm{lb} / \mathrm{A}$.

\begin{tabular}{|c|c|c|c|c|c|c|}
\hline \multicolumn{7}{|c|}{$\mathbf{P}$ and $\mathrm{K}$ index and fertilizer rate ${ }^{1}$} \\
\hline$P$ index & 3 & 6 & 9 & 12 & 15 & 18 \\
\hline $\mathrm{P}_{2} \mathrm{O}_{5}(\mathrm{Ib} / \mathrm{A})$ & 160 & 120 & 80 & 40 & 0 & 0 \\
\hline$K$ index & 50 & 80 & 110 & 140 & & \\
\hline $\mathrm{K}_{2} \mathrm{O}(\mathrm{Ib} / \mathrm{A})$ & 120 & 60 & 0 & 0 & & \\
\hline
\end{tabular}

Table 6. Plant tissue analysis for sweet corn plants 30 inches tall. Dry wt. basis.

\begin{tabular}{|c|c|c|c|c|c|c|c|c|c|c|c|c|}
\hline \multirow[b]{2}{*}{ Status } & $\mathbf{N}$ & $\mathbf{P}$ & K & $\mathrm{Ca}$ & $\mathrm{Mg}$ & $S$ & $\mathrm{Fe}$ & $\mathrm{Mn}$ & $\mathrm{Zn}$ & B & $\mathrm{Cu}$ & Mo \\
\hline & \multicolumn{6}{|c|}{ Percent } & \multicolumn{6}{|c|}{ Parts per million } \\
\hline Deficient & $<2.5$ & 0.2 & 2.5 & 0.5 & 0.2 & 0.2 & 40 & 40 & 25 & 10 & 4 & 0.1 \\
\hline Adequate range & $2.5-4.0$ & $0.2-0.4$ & $2.5-4.0$ & $0.5-0.8$ & $0.2-0.4$ & $0.2-0.4$ & $40-100$ & $40-100$ & $25-40$ & $10-30$ & $4-10$ & $0.1-0.2$ \\
\hline High & $>4.0$ & 0.4 & 4.0 & 0.8 & 0.4 & 0.4 & 100 & 100 & 40 & 30 & 10 & 0.2 \\
\hline Toxic (>) & & & & & & & & & 100 & & & \\
\hline
\end{tabular}

Table 7. Chemical weed controls: sweet corn.

\begin{tabular}{|c|c|c|c|c|}
\hline \multirow[b]{2}{*}{ Herbicide } & \multirow[b]{2}{*}{ Labeled crops } & \multirow{2}{*}{$\begin{array}{l}\text { Time of } \\
\text { application to crop }\end{array}$} & \multicolumn{2}{|c|}{ Rate (Ibs. Al./Acre) } \\
\hline & & & Mineral & Muck \\
\hline $\begin{array}{l}\text { Atrazine } \\
\text { (AAtrex } 4 \mathrm{~L}) \\
\text { (AAtrex Nine-0) }\end{array}$ & Sweet corn & Preemergence & $1.0-2.0$ & $2.0-3.0$ \\
\hline \multicolumn{5}{|c|}{$\begin{array}{l}\text { Remarks: Controls germinating annuals. Apply to moist soil. Note label precautions of planting non-registered or sensitive crops for at } \\
\text { least one growing season. }\end{array}$} \\
\hline \begin{tabular}{|l} 
Atrazine \\
(AAtrex $4 \mathrm{~L})$ \\
(AAtrex Nine-0)
\end{tabular} & Sweet corn & Postemergence & $1.0-2.8$ & $1.0-2.8$ \\
\hline \multicolumn{5}{|c|}{$\begin{array}{l}\text { Remarks: Controls emerged weeds. Apply in a minimum of } 10 \text { gals. of water before weeds are } 1.5 \text { inches tall. Use lower rates when } \\
\text { weeds are small. Note replanting precautions listed above. }\end{array}$} \\
\hline Atrazine + Oil & Sweet corn & Postemergence & $\overline{c 1.0-2.0+\text { oil }}$ & $1.0-2.0+$ oil \\
\hline \multicolumn{5}{|c|}{$\begin{array}{l}\text { Remarks: Controls emerged weeds. Apply to small test plots to evaluate tolerance of new hybrid corn varieties. Following mixing instruc- } \\
\text { tions listed on the label and rates of emulsifiable oil or oil concentrate depending on ground or aerial application methods. Apply before } \\
\text { annual grasses are } 1.50 \text { and broadleaf weeds are } 40 \text {. Note replanting precautions. Do not apply to breeding stock or inbred lines of sweet } \\
\text { corn. }\end{array}$} \\
\hline $\begin{array}{l}\text { Bentazon } \\
\text { (Basagran) }\end{array}$ & Corn (all types) & Postemergence & $0.75-1.0$ & $0.75-1.0$ \\
\hline \multicolumn{5}{|c|}{$\begin{array}{l}\text { Remarks: Controls actively growing young broadleaf weeds. Recommended for burn down of annual morning glory and yellow nutsedge } \\
\text { in corn. Consult label for weeds controlled/weed size table. Corn is tolerant at all stages of growth. Do not apply over } 2 \mathrm{lbs} \text { ai (4 pts.)/acre } \\
\text { per season. Add a crop oil concentrate ( } \operatorname{coc} \text { ) at } 2 \text { pts/acre maximum. }\end{array}$} \\
\hline
\end{tabular}


Table 7. Continued.

\begin{tabular}{||lllll|}
\hline & & Time of & \multicolumn{2}{c|}{ Rate (Ibs. Al./Acre) } \\
\cline { 3 - 5 } Herbicide & Labeled crops & application to crop & Mineral & Muck \\
\hline \hline Carfentrazone (Aim) & Corn (all) & $\begin{array}{l}\text { Preplant, Preemergence, } \\
\text { Postemergence }\end{array}$ & $0.008-0.016$ & $0.008-0.016$ \\
\hline
\end{tabular}

Remarks: Controls young actively growing broadleaf weeds. May be applied 30 days before planting until corn reaches the 8 leaf collar growth stage. Rate is $0.5 \mathrm{fl} \mathrm{oz} \mathrm{product} \mathrm{per} \mathrm{acre.} \mathrm{Use} \mathrm{a} \mathrm{nonionic} \mathrm{surfactant} \mathrm{in} \mathrm{the} \mathrm{spray} \mathrm{mix.} \mathrm{Leaf} \mathrm{burn} \mathrm{or} \mathrm{speckling} \mathrm{has} \mathrm{been} \mathrm{seen} \mathrm{on} \mathrm{older}$ plants when applied over the top. Directed sprays are much safer. No yield reduction was seen in trials when leaf damage occurred. FMC states that the use is the responsibility of grower due to not being tested on all sweet corn varieties.

\begin{tabular}{|c|c|c|}
\hline $\begin{array}{ll}\text { EPTC } & \text { Potato } \\
\text { (Eptam 7E) (Eptam 10G) } & \end{array}$ & $\begin{array}{l}\text { Postemergence or early layby } \\
\text { Preplant, Dragoff, Layby }\end{array}$ & $\begin{array}{l}3.0 \\
3.0\end{array}$ \\
\hline
\end{tabular}

Remarks: Granular formulation may be applied Preplant incorporated, at Dragoff and incorporated or at Layby and incorporated into clean cultivated soil. Emulsifiable formulation should not be applied on winter and early spring potatoes. Apply only after potatoes have emerged and true leaves have formed or at layby. There is a 45-day preharvest interval for application.

\begin{tabular}{|c|c|c|}
\hline $\begin{array}{l}\text { Glyphosate } \\
\text { (Roundup, Durango } \\
\text { Touchdown, Glyphomax) }\end{array}$ & Sweet Corn & $\begin{array}{l}\text { Chemical fallow } \\
\text { Preplant, pre emergence, } \\
\text { Pre transplant }\end{array}$ \\
\hline
\end{tabular}

Remarks: Roundup, Glyphomax and Touchdown have several formulations. Check the label of each for specific labeling directions.

\begin{tabular}{llcl}
\hline \hline Halosulfuron (Sempra) & Sweet Corn & Postemergence & 0.032
\end{tabular}

(Sandea)

Remarks: Sempra may be applied over-the-top or with drop nozzles from the spike to the layby stage of corn. Applications of $2 / 30 z$ by weight (.032 lb ai) per acre broadcast may be made with a sequential treatment of $2 / 3$ oz by wt directed or semi-directed to avoid application into the whorl may be made. Avoid cultivation for 7 days after application. Excellent control of nutsedges and active on cocklebur, pigweeds, ragweed and smartweed. Will not control emerged grasses. Consult label for plantback restrictions.

\begin{tabular}{|c|c|c|c|}
\hline $\begin{array}{l}\text { Mesotrione } \\
\text { (Callisto) }\end{array}$ & Sweet Corn & $\begin{array}{l}\text { Pre emergence } \\
\text { Postemergence }\end{array}$ & $\begin{array}{l}0.188-0.24 \\
0.094\end{array}$ \\
\hline
\end{tabular}

Remarks: Apply Callisto preemergence at 6.0 to $7.7 \mathrm{fl} \mathrm{oz/} \mathrm{A.} \mathrm{May} \mathrm{be} \mathrm{tank} \mathrm{mixed} \mathrm{with} \mathrm{a} \mathrm{grass} \mathrm{herbicide} \mathrm{for} \mathrm{grass} \mathrm{control.} \mathrm{Apply} \mathrm{at} 3 \mathrm{fl} 0 \mathrm{oz} / \mathrm{A}$ postemergence. It may be tank mixed with herbicides such as atrazine, metolachlor, bentazone, etc. Check the label. Do not apply wiht a crop oil concentrate (COC), UAN, or AMS postemergence. Corn may be treated up to 30 inches tall. Do not harvest within 45 days after applicatioon. In some cultivars, transitory bleaching may occur. Intrials, yield has not been affected.

\begin{tabular}{llrl}
\hline S-Metolachlor & Sweet Corn & Pre emergence & $1.0-1.5$
\end{tabular}

(Dual Magnum)

(Dual II Magnum)

Remarks: Provides good control of annual grasses and certain broadleaf weeds. Use the lower rate on light sandy soils. Use higher rate on soils with organic matter $3 \%$ and greater. May be used as preemergence up to 4 pints (Ibs/ai) on soils with 6 to $20 \%$ organic matter. May be used as directed spray to the base of corn plants 5 inches tall until corn plants reach 40 inches in height. See Special Local Needs (24c) label for muck soils.

\begin{tabular}{llcc}
\hline \hline Paraquat & Sweet Corn & Pre emergence & $0.56-0.94$ \\
(Gramoxone Inteon) & & \\
(Firestorm) & & \\
\hline
\end{tabular}

Remarks: Controls emerged weeds. Apply prior, during, or after planting, but before corn emerges. Use a spreader.

\begin{tabular}{llll}
\hline \hline Paraquat & Sweet Corn & Directed spray & 0.25
\end{tabular}

(Gramoxone Inteon)

Remarks: Apply when corn is at least 10" tall. Arrange nozzles to spray no higher than the lower 3 inches of the corn plant. Corn plants shorter than 10" may be injured and not recover. (Corn height measured from soil surface to top of whorl.)

\begin{tabular}{|c|c|c|c|c|}
\hline $\begin{array}{l}\text { Pendimethalin (Prowl) } \\
+ \text { Atrazine (Several) }\end{array}$ & Sweet Corn & Early Postemergence & $\begin{array}{l}0.75-1.0 \\
1.0-1.5\end{array}$ & $\begin{array}{l}1.0-2.0 \\
1.0-2.0\end{array}$ \\
\hline
\end{tabular}

Remarks: In Alabama, Florida and Georgia, Prowl 3.3 EC can be applied with atrazine early postemergence. Apply from spike through 4 leaf stage but before weeds exceed 1 inch in height, except for Texas panicum which must be no larger than the 2 leaf stage. Prowl alone will not control emerged weeds. Wait at least 7-10 days befoe cultivation early postemergence treatments.

Topramezone Sweet Corn
(Impact)


Table 8. Disease management for sweet corn.

\begin{tabular}{|c|c|c|c|c|c|c|}
\hline Chemical (a.i.) & $\begin{array}{l}\text { FRAC } \\
\text { Group } 1\end{array}$ & $\frac{\text { Maximum }}{\text { Application }}$ & $\begin{array}{l}\text { Rate/Acre/ } \\
\text { Season }\end{array}$ & $\begin{array}{l}\text { Min. Days } \\
\text { to Harvest }\end{array}$ & Pertinent Diseases & Select Remarks ${ }^{2}$ \\
\hline $\begin{array}{l}\text { Amistar 80DF } \\
\text { (Azoxystrobin) }\end{array}$ & 11 & $50 z$ & $2.5 \mathrm{lb}$ & 7 & $\begin{array}{l}\text { Common and southern rust } \\
\text { Northern corn leaf blight } \\
\text { Southern corn leaf blight }\end{array}$ & $\begin{array}{l}\text { Do not exceed } 1 \text { sequential and } \\
6 \text { total applications of Amistar } \\
\text { or other Qol fungicides. }\end{array}$ \\
\hline $\begin{array}{l}\text { Apron XL LS } \\
\text { (Mefenoxam) }\end{array}$ & 4 & $\begin{array}{l}0.64 \mathrm{fl} .0 \mathrm{z} . / \\
100 \mathrm{lb} \mathrm{seed} \\
\text { or } \\
2.2 \mathrm{fl} \mathrm{oz/} \\
100 \mathrm{lb} \text { seed }\end{array}$ & & & $\begin{array}{l}\text { Pythium seedling blight } \\
\text { Downy mildew }\end{array}$ & $\begin{array}{l}\text { Seed treatment only. Use the } \\
\text { higher rate if treating seed for } \\
\text { prevention of systemic downy } \\
\text { mildew. }\end{array}$ \\
\hline $\begin{array}{l}\text { Bravo Ultrex } 82.5 \\
\text { WDG } \\
\text { (Chlorothalonil) }\end{array}$ & M5 & $1.8 \mathrm{lb}$ & $10.9 \mathrm{lb}$ & 14 & $\begin{array}{l}\text { Common and southern rust } \\
\text { Northern corn leaf blight } \\
\text { Southern corn leaf blight }\end{array}$ & $\begin{array}{l}\text { Do not use crop for livestock } \\
\text { feed. }\end{array}$ \\
\hline $\begin{array}{l}\text { Bravo Weather Stik 6F } \\
\text { (Chlorothalonil) }\end{array}$ & M5 & $2 \mathrm{pt}$ & $12 \mathrm{pt}$ & 14 & $\begin{array}{l}\text { Common and southern rust } \\
\text { Northern corn leaf blight } \\
\text { Southern corn leaf blight }\end{array}$ & $\begin{array}{l}\text { Do not use crop for livestock } \\
\text { feed. }\end{array}$ \\
\hline $\begin{array}{l}\text { Bumper 41.8EC } \\
\text { (Propiconazole) }\end{array}$ & 3 & $4 \mathrm{fl} \mathrm{oz}$ & $16 \mathrm{fl} \mathrm{oz}$ & 14 & $\begin{array}{l}\text { Common and southern rust } \\
\text { Northern corn leaf blight } \\
\text { Southern corn leaf blight }\end{array}$ & $\begin{array}{l}\text { Do not exceed } 4 \text { total applica- } \\
\text { tions. }\end{array}$ \\
\hline $\begin{array}{l}\text { Contans WG } \\
\text { (Coniothyrium mini- } \\
\text { tans) }\end{array}$ & & $6 \mathrm{lbs}$ & & & Sclerotinia diseases & $\begin{array}{l}\text { Apply to soil surface and incor- } \\
\text { porate prior to, at planting. }\end{array}$ \\
\hline $\begin{array}{l}\text { Copper-Count-N } \\
\text { (Copper ammonium } \\
\text { complex) }\end{array}$ & M1 & $2 q t$ & & & $\begin{array}{l}\text { Bacterial rot, bacterial wilt, } \\
\text { Bacterial stripe, Leaf } \\
\text { blights, Stalk rot }\end{array}$ & \\
\hline $\begin{array}{l}\text { Dithane-DF-Rainshield } \\
\text { (Mancozeb) }\end{array}$ & M3 & $1.5 \mathrm{lb}$ & $22.5 \mathrm{lb}$ & 7 & $\begin{array}{l}\text { Common and southern rust } \\
\text { Northern corn leaf blight } \\
\text { Southern corn leaf blight }\end{array}$ & $\begin{array}{l}\text { Do not use crop for livestock } \\
\text { feed. }\end{array}$ \\
\hline $\begin{array}{l}\text { Dithane-F45- } \\
\text { Rainshield } \\
\text { (Mancozeb) }\end{array}$ & M3 & $1.2 \mathrm{qt}$ & $18 \mathrm{qt}$ & 7 & $\begin{array}{l}\text { Common and southern rust } \\
\text { Northern corn leaf blight } \\
\text { Southern corn leaf blight }\end{array}$ & $\begin{array}{l}\text { Do not use crop for livestock } \\
\text { feed. }\end{array}$ \\
\hline $\begin{array}{l}\text { Dithane-M45 } \\
\text { (Mancozeb) }\end{array}$ & M3 & $1.5 \mathrm{lb}$ & $22.5 \mathrm{lb}$ & 7 & $\begin{array}{l}\text { Common and southern rust } \\
\text { Northern corn leaf blight } \\
\text { Southern corn leaf blight }\end{array}$ & $\begin{array}{l}\text { Do not use crop for livestock } \\
\text { feed. }\end{array}$ \\
\hline $\begin{array}{l}\text { Echo } 720 \\
\text { (Chlorothalonil) }\end{array}$ & M5 & $2 \mathrm{pt}$ & $12 \mathrm{pt}$ & 14 & $\begin{array}{l}\text { Common and southern rust } \\
\text { Northern corn leaf blight } \\
\text { Southern corn leaf blight }\end{array}$ & $\begin{array}{l}\text { Do not use crop for livestock } \\
\text { feed. }\end{array}$ \\
\hline $\begin{array}{l}\text { Echo } 90 \text { DF } \\
\text { (Chlorothalonil) }\end{array}$ & M5 & $1.6 \mathrm{lb}$ & $10 \mathrm{lb}$ & 14 & $\begin{array}{l}\text { Common and southern rust } \\
\text { Northern corn leaf blight } \\
\text { Southern corn leaf blight }\end{array}$ & $\begin{array}{l}\text { Do not use crop for livestock } \\
\text { feed. }\end{array}$ \\
\hline $\begin{array}{l}\text { Equus } 720 \text { SST } \\
\text { (Chlorothalonil) }\end{array}$ & M5 & $2 \mathrm{pt}$ & $12 \mathrm{pt}$ & 14 & $\begin{array}{l}\text { Common and southern rust } \\
\text { Northern corn leaf blight } \\
\text { Southern corn leaf blight }\end{array}$ & $\begin{array}{l}\text { Do not use crop for livestock } \\
\text { feed. }\end{array}$ \\
\hline $\begin{array}{l}\text { Equus-DF } \\
\text { (Chlorothalonil) }\end{array}$ & M5 & $1.6 \mathrm{lb}$ & $10 \mathrm{lb}$ & 14 & $\begin{array}{l}\text { Common and southern rust } \\
\text { Northern corn leaf blight } \\
\text { Southern corn leaf blight }\end{array}$ & $\begin{array}{l}\text { Do not use crop for livestock } \\
\text { feed. }\end{array}$ \\
\hline $\begin{array}{l}\text { Fosphite } \\
\text { (Potassium phos- } \\
\text { phite) }\end{array}$ & 33 & $3 \mathrm{qt}$ & $18 \mathrm{qt}$ & & $\begin{array}{l}\text { Pythium, Rhizoctonia, } \\
\text { Fusarium, Downy mildew }\end{array}$ & $\begin{array}{l}\text { Do not exceed } 6 \text { applications } \\
\text { per crop. Caution should be } \\
\text { used when applying in a man- } \\
\text { agement program including } \\
\text { copper fungicides. See label for } \\
\text { foliar, and irrigation application } \\
\text { details. }\end{array}$ \\
\hline $\begin{array}{l}\text { Headline EC } \\
\text { (Pyraclostrobin) }\end{array}$ & 11 & $12 \mathrm{fl} \mathrm{oz}$ & $72 \mathrm{fl} \mathrm{oz}$ & 7 & $\begin{array}{l}\text { Common and southern rust } \\
\text { Northern corn leaf blight } \\
\text { Southern corn leaf blight }\end{array}$ & $\begin{array}{l}\text { Do not exceed } 2 \text { sequential and } \\
6 \text { total applications of Headline } \\
\text { or other Qol fungicides. }\end{array}$ \\
\hline
\end{tabular}


Table 8. Continued.

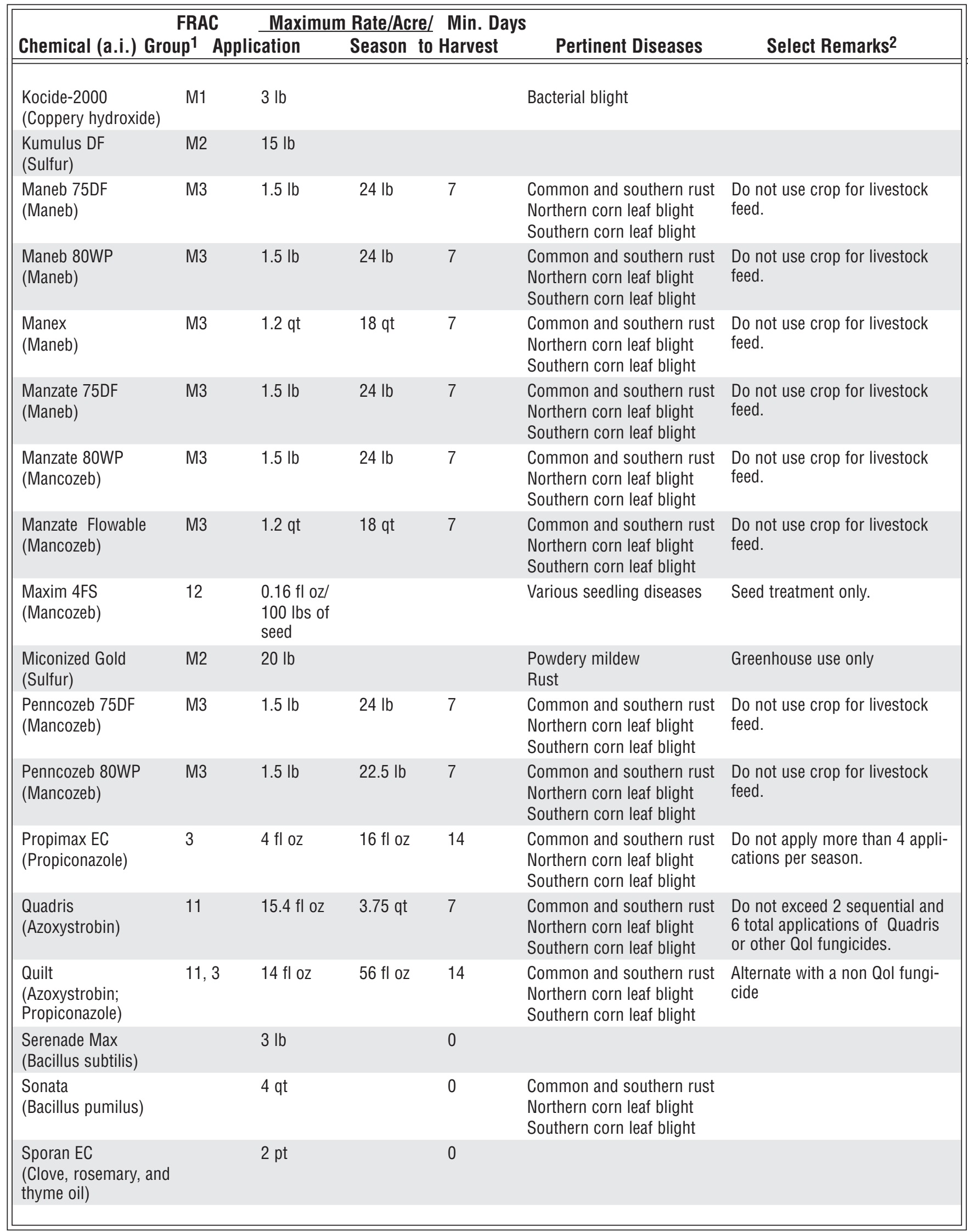


Table 8. Continued.

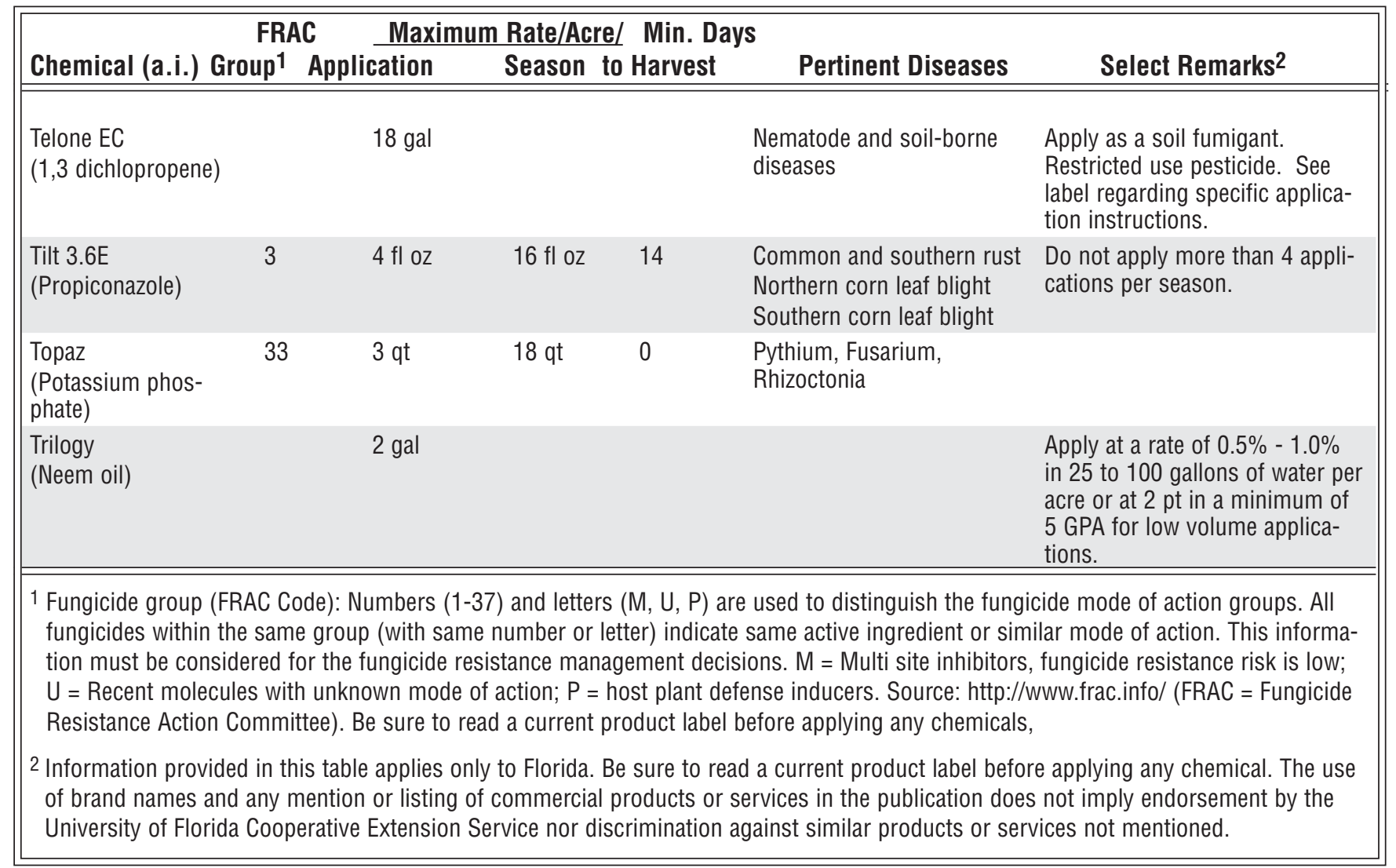


Table 9. Selected insecticides approved for use on insects attacking sweet corn.

\begin{tabular}{|c|c|c|c|c|c|c|}
\hline $\begin{array}{l}\text { Trade Name } \\
\text { (Common Name) }\end{array}$ & $\begin{array}{l}\text { Rate } \\
\text { (product/acre) }\end{array}$ & $\begin{array}{l}\text { REI } \\
\text { (hours) }\end{array}$ & $\begin{array}{l}\text { Days to } \\
\text { Harvest }\end{array}$ & Insects & $\begin{array}{l}\text { MOA } \\
\text { Code }^{1}\end{array}$ & Notes \\
\hline $\begin{array}{l}\text { Agree WG } \\
\text { (Bacillus thuringiensis } \\
\text { subspecies aizawai) }\end{array}$ & $1.0-2.0 \mathrm{lb}$ & 4 & 0 & $\begin{array}{l}\text { lepidopteran larvae (cater- } \\
\text { pillar pests) }\end{array}$ & $11 \mathrm{~B} 1$ & $\begin{array}{l}\text { Apply when larvae are small for } \\
\text { best control. OMRI-listed?2. }\end{array}$ \\
\hline $\begin{array}{l}\text { *Ambush 25W } \\
\text { (permethrin) }\end{array}$ & $6.4-16 \mathrm{oz}$ & 12 & 1 & $\begin{array}{l}\text { aster leafhopper, corn ear- } \\
\text { worm, corn rootworm } \\
\text { (adults), cutworms, fall } \\
\text { armyworm }\end{array}$ & 3 & $\begin{array}{l}\text { Do not apply more than } 2.0 \text { ai/ } \\
\text { acre per season. (128 oz) }\end{array}$ \\
\hline $\begin{array}{l}\text { *Asana XL (0.66EC) } \\
\text { (esfenvalerate) }\end{array}$ & $5.8-9.6 \mathrm{fl} \mathrm{oz}$ & 12 & 1 & $\begin{array}{l}\text { aphids, armyworms, } \\
\text { banded cucumber beetle, } \\
\text { beet armyworm (aids in } \\
\text { control), chinch bugs, } \\
\text { corn borer, corn earworm, } \\
\text { corn rootworm, corn silk } \\
\text { fly, cutworms, flea beetles, } \\
\text { grasshoppers, sap beetle } \\
\text { (adults), stalkborers, tar- } \\
\text { nished plant bug }\end{array}$ & 3 & $\begin{array}{l}\text { Do not apply more than } 0.5 \mathrm{lb} \text { ai/ } \\
\text { acre per season (10 applications } \\
\text { at highest rate). }\end{array}$ \\
\hline $\begin{array}{l}\text { Avaunt } \\
\text { (indoxacarb) }\end{array}$ & 2.5-3.5 oz & $\begin{array}{l}12,(14 \\
\text { days for } \\
\text { hand } \\
\text { harvest- } \\
\text { ing) }\end{array}$ & $\begin{array}{l}\text { 3, ( } 35 \\
\text { for fod- } \\
\text { der \& } \\
\text { stover) }\end{array}$ & fall armyworm & 22 & $\begin{array}{l}\text { Whorl application (before silking) } \\
\text { only. No more than } 4 \text { applications } \\
\text { per season. }\end{array}$ \\
\hline $\begin{array}{l}\text { Aza-Direct (azadi- } \\
\text { rachtin) }\end{array}$ & $\begin{array}{l}1-2 \mathrm{pts} \text {, up } \\
\text { to } 3.5 \mathrm{pts} \text {, if } \\
\text { needed }\end{array}$ & 4 & 0 & $\begin{array}{l}\text { aphids, beetles, caterpil- } \\
\text { lars, leafhoppers, leafmin- } \\
\text { ers, mites, stink bugs, } \\
\text { thrips, weevils, whiteflies }\end{array}$ & 26 & $\begin{array}{l}\text { Antifeedant, repellant, insect } \\
\text { growth regulator. OMRI-listed². }\end{array}$ \\
\hline $\begin{array}{l}\text { Azatin XL } \\
\text { (azadirachtin) }\end{array}$ & $5-21 \mathrm{fl} \mathrm{oz}$ & 4 & 0 & $\begin{array}{l}\text { aphids, beetles, caterpil- } \\
\text { lars, leafhoppers, leafmin- } \\
\text { ers, thrips, weevils, } \\
\text { whiteflies }\end{array}$ & 26 & $\begin{array}{l}\text { Antifeedant, repellant, insect } \\
\text { growth regulator. }\end{array}$ \\
\hline $\begin{array}{l}\text { *Baythroid } 2 \\
\text { (cyfluthrin) }\end{array}$ & $0.8-2.8 \mathrm{fl} \mathrm{oz}$ & 12 & 0 & $\begin{array}{l}\text { chinch bugs, common } \\
\text { stalk borers, corn ear- } \\
\text { worm, corn rootworm } \\
\text { adult, corn silk fly, cut- } \\
\text { worms, fall armyworm } \\
\text { (1st and 2nd instars only), } \\
\text { grasshoppers, true army- } \\
\text { worm }\end{array}$ & 3 & $\begin{array}{l}\text { Maximum number of applica- } \\
\text { tions: } 10 \text {. }\end{array}$ \\
\hline $\begin{array}{l}\text { Biobit HP } \\
\text { (Bacillus thuringiensis } \\
\text { subspecies kurstaki) }\end{array}$ & $0.5-2.0 \mathrm{lb}$ & 4 & 0 & $\begin{array}{l}\text { caterpillars (will not con- } \\
\text { trol large armyworms) }\end{array}$ & 11B2 & $\begin{array}{l}\text { Treat when larvae are young. } \\
\text { Good coverage is essential. } \\
\text { Can be used in the greenhouse. } \\
\text { OMRI-listed?2. }^{2}\end{array}$ \\
\hline $\begin{array}{l}\text { BotaniGard } 22 \text { WP, ES } \\
\text { (Beauveria bassiana) }\end{array}$ & $\begin{array}{l}\text { WP: } \\
0.5-2 \mathrm{lb} / 100 \mathrm{gal} \\
\text { ES: } \\
0.5-2 \text { qts/100 } \\
\text { gal }\end{array}$ & 4 & 0 & aphids, thrips, whiteflies & -- & $\begin{array}{l}\text { May be used in greenhouses. } \\
\text { Contact dealer for recommenda- } \\
\text { tions if an adjuvant must be used. } \\
\text { Not compatible in tank mix with } \\
\text { fungicides. }\end{array}$ \\
\hline
\end{tabular}


Table 9. Continued.

\begin{tabular}{|c|c|c|c|c|c|c|}
\hline $\begin{array}{l}\text { Trade Name } \\
\text { (Common Name) }\end{array}$ & $\begin{array}{l}\text { Rate } \\
\text { (product/acre) }\end{array}$ & $\begin{array}{l}\text { REI } \\
\text { (hours) }\end{array}$ & $\begin{array}{l}\text { Days to } \\
\text { Harvest }\end{array}$ & Insects & $\begin{array}{l}\text { MOA } \\
\text { Code }^{1}\end{array}$ & Notes \\
\hline $\begin{array}{l}{ }^{*} \text { Counter 15G } \\
\text { Lock 'n Load } \\
\text { (terbufos) }\end{array}$ & $\begin{array}{l}6.0-8.0 \text { oz per } \\
1000 \mathrm{ft} \text { of row, } \\
\text { banded or in } \\
\text { furrow } \\
\text { post emergence } \\
\text { incorporated, } 8 \\
\text { 0z per } 1000 \mathrm{ft} \\
\text { of row at culti- } \\
\text { vation, } 8 \mathrm{oz} \mathrm{per} \\
1000 \mathrm{ft} \text { of row } \\
\text { at cultivation, } 8 \\
\text { 0z per } 1000 \mathrm{ft} \\
\text { of row }\end{array}$ & 48 & 60 & $\begin{array}{l}\text { billbugs, chinch bugs }(1) \text {, } \\
\text { corn rootworm, cut- } \\
\text { worms (suppression), } \\
\text { flea beetles, lesser corn } \\
\text { stalk borer (suppression), } \\
\text { maize billbug, seedcorn } \\
\text { beetle, seedcorn maggot, } \\
\text { symphylans, thrips, white } \\
\text { grubs, wireworms }\end{array}$ & $1 \mathrm{~B}$ & $\begin{array}{l}(1) \text { Early season control of light } \\
\text { to moderate infestations. Only } \\
\text { one application (at-planting, post- } \\
\text { emergence incorporated, or culti- } \\
\text { vation time treatment per season. } \\
\text { Do not exceed } 8.7 \mathrm{lb} / \text { acre. }\end{array}$ \\
\hline $\begin{array}{l}\text { Crymax WDG } \\
\text { (Bacillus thuringiensis } \\
\text { subspecies kurstaki) }\end{array}$ & $0.5-2.0 \mathrm{lb}$ & 4 & 0 & caterpillars & $11 \mathrm{~B} 2$ & $\begin{array}{l}\text { Use high rate for armyworms. } \\
\text { Treat when larvae are young. }\end{array}$ \\
\hline $\begin{array}{l}\text { Deliver } \\
\text { (Bacillus thuringiensis } \\
\text { subspecies kurstaki) }\end{array}$ & $0.25-1.5 \mathrm{lb}$ & 4 & 0 & caterpillars & 11B2 & $\begin{array}{l}\text { Use higher rates for armyworms. } \\
\text { OMRI-listed? }\end{array}$ \\
\hline $\begin{array}{l}\text { *Diazinon 4EC, *50W } \\
\text { (diazinon) }\end{array}$ & $\begin{array}{l}\text { foliar - } \\
\text { AG500, 4EC: } \\
0.5-2.5 \text { pts } \\
\text { 50W: } 1-2.5 \mathrm{lb} \\
\\
\text { preplant - } \\
\text { AG500, 4EC: } \\
\text { 2-4 qts } \\
\text { 50W: } 4-8 \mathrm{lb}\end{array}$ & 24 & $\begin{array}{l}7 \\
\text { or } \\
\text { preplant }\end{array}$ & $\begin{array}{l}\text { corn earworm, corn leaf } \\
\text { aphid, corn rootworm } \\
\text { adult, cutworms, flea } \\
\text { beetles, grasshoppers, } \\
\text { sap beetles, seed corn } \\
\text { maggot, spider mites, } \\
\text { wireworms }\end{array}$ & $1 \mathrm{~B}$ & $\begin{array}{l}\text { Apply just before planting for } \\
\text { seed corn maggot, cutworms, } \\
\text { and wireworms. Do not make } \\
\text { more than } 5 \text { applications per sea- } \\
\text { son (4E). }\end{array}$ \\
\hline $\begin{array}{l}\text { DiPel DF } \\
\text { (Bacillus thuringiensis } \\
\text { subspecies kurstaki) }\end{array}$ & $0.5-2.0 \mathrm{lb}$ & 4 & 0 & caterpillars & $11 \mathrm{~B} 2$ & $\begin{array}{l}\text { Treat when larvae are young. } \\
\text { Good coverage is essential. } \\
\text { OMRI-listed? }\end{array}$ \\
\hline $\begin{array}{l}\text { Endosulfan 3EC (endo- } \\
\text { sulfan) }\end{array}$ & $1.33-2 \mathrm{qt}$ & 24 & 1 & $\begin{array}{l}\text { aphids, corn earworm, } \\
\text { whiteflies }\end{array}$ & 2 & $\begin{array}{l}\text { Do not apply to sweet corn to } \\
\text { be processed or used to feed } \\
\text { livestock. }\end{array}$ \\
\hline $\begin{array}{l}\text { Entrust } \\
\text { (spinosad) }\end{array}$ & $0.5-20 z$ & 4 & $\begin{array}{l}1 \text { day } \\
\text { - ears } \\
7 \text { day - } \\
\text { forage }\end{array}$ & $\begin{array}{l}\text { armyworms, corn ear- } \\
\text { worm }\end{array}$ & 5 & $\begin{array}{l}\text { Do not apply more than } 9 \text { oz per } \\
\text { acre per year. } \\
\text { OMRI-listed }{ }^{2} \text {. }\end{array}$ \\
\hline $\begin{array}{l}\text { Extinguish } \\
((\mathrm{S}) \text {-methoprene) }\end{array}$ & $1-1.5 \mathrm{lb}$ & 4 & 0 & fire ants & $7 \mathrm{~A}$ & $\begin{array}{l}\text { Slow-acting IGR (insect growth } \\
\text { regulator). Best applied early } \\
\text { spring and fall where crop will be } \\
\text { grown. Colonies will be reduced } \\
\text { after three weeks and eliminated } \\
\text { after } 8 \text { to } 10 \text { weeks. May be } \\
\text { applied by ground equipment or } \\
\text { aerially. }\end{array}$ \\
\hline $\begin{array}{l}\text { *Force } 3 \mathbf{G} \\
\text { (tefluthrin) }\end{array}$ & $\begin{array}{l}\text { depends on row } \\
\text { spacing }\end{array}$ & 0 & $\begin{array}{l}\text { at plant- } \\
\text { ing or } \\
\text { cultiva- } \\
\text { tion }\end{array}$ & $\begin{array}{l}\text { billbugs }(1) \text {, chinch bugs }(1) \text {, } \\
\text { corn rootworm, cut- } \\
\text { worms, lesser cornstalk } \\
\text { borer, red imported fire }\end{array}$ & 3 & $\begin{array}{l}\text { Only one application per crop. } \\
\text { Granules must be } \\
\text { incorporated. } \\
\text { (1) suppression only }\end{array}$ \\
\hline & & & $\begin{array}{l}\text { Withing } 30 \\
\text { days of } \\
\text { seeding } \\
\text { emer- } \\
\text { gence }\end{array}$ & $\begin{array}{l}\text { seedcorn maggot, white } \\
\text { grubs, wireworms }\end{array}$ & & (2) suppression for 28 days \\
\hline
\end{tabular}


Table 9. Continued.

\begin{tabular}{|c|c|c|c|c|c|c|}
\hline $\begin{array}{l}\text { Trade Name } \\
\text { (Common Name) }\end{array}$ & $\begin{array}{l}\text { Rate } \\
\text { (product/acre) }\end{array}$ & $\begin{array}{l}\text { REI } \\
\text { (hours) }\end{array}$ & $\begin{array}{l}\text { Days to } \\
\text { Harvest }\end{array}$ & Insects & $\begin{array}{l}\text { MOA } \\
\text { Code1 }\end{array}$ & Notes \\
\hline $\begin{array}{l}{ }^{*} \text { Furadan } 4 \mathrm{~F} \text { (carbo- } \\
\text { furan) }\end{array}$ & $2.5 \mathrm{oz} / 1000 \mathrm{ft}$ & 48 & $\begin{array}{l}\text { at plant- } \\
\text { ing }\end{array}$ & $\begin{array}{l}\text { corn rootworms, flea } \\
\text { beetles, seedcorn maggot, } \\
\text { wireworms }\end{array}$ & $1 \mathrm{~A}$ & $\begin{array}{l}\text { See restrictions for very sandy } \\
\text { soil. }\end{array}$ \\
\hline $\begin{array}{l}\text { Javelin WG } \\
\text { (Bacillus thuringiensis } \\
\text { subspecies kurstaki) }\end{array}$ & $0.12-1.50 \mathrm{lb}$ & 4 & 0 & $\begin{array}{l}\text { most caterpillars, but } \\
\text { not Spodoptera species } \\
\text { (armyworms) }\end{array}$ & $11 \mathrm{~B} 2$ & $\begin{array}{l}\text { Treat when larvae are young. } \\
\text { Thorough coverage is essential. } \\
\text { OMRI-listed². }^{2}\end{array}$ \\
\hline $\begin{array}{l}\text { *Lannate LV, *SP } \\
\text { (methomyl) }\end{array}$ & $\begin{array}{l}\text { LV: } \\
0.75-1.5 \mathrm{pts} \\
\text { SP: } \\
0.25-0.50 \mathrm{lb}\end{array}$ & 48 & $\begin{array}{l}0 \text { - ears } \\
3 \text { - for- } \\
\text { age } \\
21 \text { - sto- } \\
\text { ver }\end{array}$ & $\begin{array}{l}\text { aphids, armyworms, } \\
\text { beet armyworm, corn } \\
\text { earworm, corn rootworm, } \\
\text { cutworms, fall armyworm, } \\
\text { flea beetles, picnic beetles }\end{array}$ & $1 \mathrm{~A}$ & $\begin{array}{l}\text { Certain hybrid varieties are sus- } \\
\text { ceptible to methomyl injury. Treat } \\
\text { a small area to determine safety } \\
\text { first. }\end{array}$ \\
\hline $\begin{array}{l}\text { *Larvin } 3.2 \\
\text { (thiodicarb) }\end{array}$ & $20-30 \mathrm{fl} \mathrm{oz}$ & 48 & 0 & $\begin{array}{l}\text { beet armyworm, cut- } \\
\text { worms, corn earworm, } \\
\text { fall armyworm, southern } \\
\text { armyworm }\end{array}$ & $1 \mathrm{~A}$ & $\begin{array}{l}\text { Do not allow livestock to graze } \\
\text { treated field. Do not feed treated } \\
\text { corn silage or fodder to livestock } \\
\text { See label for special instructions } \\
\text { for cutworms. }\end{array}$ \\
\hline $\begin{array}{l}\text { Lepinox WDG } \\
\text { (Bacillus thuringiensis } \\
\text { subspecies kurstaki) }\end{array}$ & $1.0-2.0 \mathrm{lb}$ & 12 & 0 & $\begin{array}{l}\text { for most caterpillars, } \\
\text { including beet armyworm } \\
\text { (see label) }\end{array}$ & $11 \mathrm{~B} 2$ & $\begin{array}{l}\text { Treat when larvae are small. } \\
\text { Thorough coverage is essential. }\end{array}$ \\
\hline $\begin{array}{l}\text { *Lorsban } 75 W G \text { (chlor- } \\
\text { pyrifos) }\end{array}$ & $0.33-1.33 \mathrm{lb}$ & 24 & 35 & $\begin{array}{l}\text { aphids, beet armyworm, } \\
\text { chinch bugs, corn ear- } \\
\text { worm, corn rootworm } \\
\text { adult, cutworms, fall } \\
\text { armyworm, grasshoppers }\end{array}$ & $1 \mathrm{~B}$ & $\begin{array}{l}\text { Do not feed treated corn silage, } \\
\text { forage, or fodder, or allow live- } \\
\text { stock to graze. }\end{array}$ \\
\hline 15G, 75 WG & $\begin{array}{l}\text { See label for } \\
\text { rates. }\end{array}$ & 24 & $\begin{array}{l}\text { at plant- } \\
\text { ing }\end{array}$ & $\begin{array}{l}\text { billbugs, corn rootworm } \\
\text { larvae, cutworms, lesser } \\
\text { corn stalk borer, seed } \\
\text { corn maggot, symphylans, } \\
\text { wireworms }\end{array}$ & 1B & See label. \\
\hline $\begin{array}{l}\text { *MSR Spray } \\
\text { Concentrate (oxy- } \\
\text { demeton-methyl) }\end{array}$ & $1.5-2 \mathrm{pt}$ & 48 & $\begin{array}{l}7-1 \\
\text { appl } \\
21-2 \\
\text { or } 3 \\
\text { applica- } \\
\text { tions }\end{array}$ & $\begin{array}{l}\text { aphids, corn rootworms, } \\
\text { leafhoppers, mites, thrips }\end{array}$ & $1 \mathrm{~B}$ & $\begin{array}{l}\text { Do not apply more than } 3 \text { times } \\
\text { per season. }\end{array}$ \\
\hline $\begin{array}{l}\text { *Mocap 15G, *EC } \\
\text { (ethoprop) }\end{array}$ & See label. & 48 & $\begin{array}{l}\text { at plant- } \\
\text { ing }\end{array}$ & $\begin{array}{l}\text { corn rootworms, cut- } \\
\text { worms, symphylans, } \\
\text { wireworms, (suppression } \\
\text { of white grubs) }\end{array}$ & $1 \mathrm{~B}$ & \\
\hline $\begin{array}{l}\text { M-Pede } \mathbf{4 9 \%} \text { EC } \\
\text { Soap, Insecticidal }\end{array}$ & $1-2 \%$ VN & 12 & 0 & $\begin{array}{l}\text { aphids, armyworms, leaf- } \\
\text { hoppers, mites, thrips }\end{array}$ & -- & OMRI-listed². \\
\hline $\begin{array}{l}{ }^{*} \text { Mustang Max } \\
\text { (zeta-cypermethrin) }\end{array}$ & $2.24-4.0 \mathrm{oz}$ & 12 & 3 & $\begin{array}{l}\text { armyworms, chinch } \\
\text { bug, corn borers, corn } \\
\text { earworm, corn silkfly, } \\
\text { cutworms, flea beetles, } \\
\text { grasshoppers, leafhop- } \\
\text { pers, sap beetle adults }\end{array}$ & 3 & $\begin{array}{l}\text { Maximum of } 0.15 \mathrm{lb} \text { ai/acre per } \\
\text { season. }\end{array}$ \\
\hline $\begin{array}{l}\text { Neemix } 4.5 \\
\text { (azadirachtin) }\end{array}$ & $4-16 \mathrm{fl} \mathrm{oz}$ & 12 & 0 & $\begin{array}{l}\text { aphids, armyworms, corn } \\
\text { earworm, thrips }\end{array}$ & 26 & OMRI-listed². \\
\hline Oil, insecticidal & $\begin{array}{l}1-2 \text { gal } / 100 \mathrm{gal} \text {, } \\
\text { depending on } \\
\text { brand }\end{array}$ & 4 & 0 & $\begin{array}{l}\text { aphids, armyworms, corn } \\
\text { earworms, corn root- } \\
\text { worms, mites, thrips }\end{array}$ & -- & \\
\hline
\end{tabular}


Table 9. Continued.

\begin{tabular}{|c|c|c|c|c|c|c|}
\hline $\begin{array}{l}\text { Trade Name } \\
\text { (Common Name) }\end{array}$ & $\begin{array}{l}\text { Rate } \\
\text { (product/acre) }\end{array}$ & $\begin{array}{l}\text { REI } \\
\text { (hours) }\end{array}$ & $\begin{array}{l}\text { Days to } \\
\text { Harvest I }\end{array}$ & Insects & $\begin{array}{l}\text { MOA } \\
\text { Code }^{1}\end{array}$ & Notes \\
\hline $\begin{array}{l}\text { *Penncap-M 2EC } \\
\text { (methyl parathion) }\end{array}$ & $1-3 \mathrm{pt}$ & $\begin{array}{l}4 \text { days } \\
\text { - See } \\
\text { label }\end{array}$ & 4 & $\begin{array}{l}\text { aphids, armyworms, corn } \\
\text { earworm, corn rootworm } \\
\text { adult, cutworms, flea } \\
\text { beetles, grasshoppers, } \\
\text { sap beetles, silk fly }\end{array}$ & $1 \mathrm{~B}$ & See restrictions on label. \\
\hline \multirow[t]{3}{*}{$\begin{array}{l}\text { *Pounce } 1.5 \mathrm{G} \\
\text { (permethrin) }\end{array}$} & $80 z / 1000 \mathrm{ft}$ & 12 & $\begin{array}{l}\text { at plant- } \\
\text { ing }\end{array}$ & armyworms, cutworms & 3 & \\
\hline & $6.7-13.3 \mathrm{lb}$ & & $\begin{array}{l}\text { pr } \\
\text { e-emer- } \\
\text { gence }\end{array}$ & $\begin{array}{l}\text { armyworms, cutworms, } \\
\text { stalk borers }\end{array}$ & 3 & \\
\hline & $6.7-13.3 \mathrm{lb}$ & & foliar - 1 & $\begin{array}{l}\text { armyworms, corn borers, } \\
\text { cutworms, stalk borers }\end{array}$ & 3 & \\
\hline $\begin{array}{l}\text { Pyrellin EC (pyrethrin + } \\
\text { rotenone) }\end{array}$ & $1-2 \mathrm{pt}$ & 12 & 12 hours & $\begin{array}{l}\text { aphids, flea beetles, leaf- } \\
\text { hoppers, loopers, mites, } \\
\text { thrips }\end{array}$ & 3,21 & \\
\hline $\begin{array}{l}\text { Sevin 80S; 4F; XLR } \\
\text { (carbaryl) }\end{array}$ & $\begin{array}{l}\text { 80S: } 1.25-2.5 \mathrm{lb} \\
\text { 4F; XLR: } 1-2 \text { qt }\end{array}$ & 12 & $\begin{array}{l}2 \text { - Ears } \\
14 \\
- \text { Forage } \\
48- \\
\text { Fodder }\end{array}$ & $\begin{array}{l}\text { armyworms, chinchbugs, } \\
\text { corn earworms, corn root- } \\
\text { worm adult, cutworms, } \\
\text { fall armyworm, flea } \\
\text { beetles, leafhoppers, sap } \\
\text { beetles }\end{array}$ & $1 \mathrm{~A}$ & Highly toxic to bees. \\
\hline $\begin{array}{l}\text { SpinTor } 2 \text { SC } \\
\text { (spinosad) }\end{array}$ & $1.5-6 \mathrm{fl} \mathrm{oz}$ & 4 & 1 & $\begin{array}{l}\text { armyworms, corn ear- } \\
\text { worm }\end{array}$ & 5 & $\begin{array}{l}\text { Do not apply more than } 29 \mathrm{fl} \mathrm{oz} \\
\text { per acre per year. }\end{array}$ \\
\hline $\begin{array}{l}\text { *Telone C-35 (dichloro- } \\
\text { propene + chloropicrin) } \\
{ }^{*} \text { Telone II } \\
\text { (dichloropropene) }\end{array}$ & See label. & $\begin{array}{l}5 \text { days } \\
- \text { See } \\
\text { label }\end{array}$ & preplant & symphylans, wireworms & -- & $\begin{array}{l}\text { See supplemental label for use } \\
\text { restrictions in south and central } \\
\text { Florida. }\end{array}$ \\
\hline $\begin{array}{l}\text { *Thimet 20-G } \\
\text { (phorate) }\end{array}$ & $\begin{array}{l}\text { See label. No } \\
\text { more than } 6.5 \\
\text { lb }\end{array}$ & 48 & $\begin{array}{l}\text { at plant- } \\
\text { ing, see } \\
\text { label }\end{array}$ & $\begin{array}{l}\text { corn rootworms, flea } \\
\text { beetles, mites, seedcorn } \\
\text { beetle, seed corn maggot, } \\
\text { white grubs, wireworms }\end{array}$ & 1B & One application per season. \\
\hline $\begin{array}{l}\text { Trilogy } \\
\text { (extract of neem oil) }\end{array}$ & $0.5-2.0 \% \mathrm{~V} / \mathrm{V}$ & 4 & 0 & $\begin{array}{l}\text { aphids, mites, suppres- } \\
\text { sion of thrips and white- } \\
\text { flies }\end{array}$ & 26 & $\begin{array}{l}\text { Apply morning or evening to } \\
\text { reduce potential for leaf burn. } \\
\text { Toxic to bees exposed to direct } \\
\text { treatment. } \\
\text { OMRI-listed2. }\end{array}$ \\
\hline $\begin{array}{l}{ }^{*} \text { Warrior (lambda-cyh- } \\
\text { alothrin) }\end{array}$ & $2.56-3.84 \mathrm{fl} \mathrm{oz}$ & 24 & $\begin{array}{l}1 \\
21 \text { for } \\
\text { feeding } \\
\text { livestock }\end{array}$ & $\begin{array}{l}\text { aphids, aster leafhopper, } \\
\text { beet armyworm, chinch } \\
\text { bugs, corn earworm, corn } \\
\text { rootworm, cutworms, fall } \\
\text { armyworm, flea beetles, } \\
\text { grasshoppers, mites (see } \\
\text { label for more details), red } \\
\text { imported fire ant( }(1), \text { south- } \\
\text { ern armyworm, tarnished } \\
\text { plant bug, wireworm(1) }\end{array}$ & 3 & (1) suppression only. \\
\hline
\end{tabular}


Table 9. Continued.

\begin{tabular}{||lllllll|}
\hline $\begin{array}{l}\text { Trade Name } \\
\text { (Common Name) }\end{array}$ & $\begin{array}{l}\text { Rate } \\
\text { (product/acre) }\end{array}$ & $\begin{array}{l}\text { REI } \\
\text { (hours) }\end{array}$ & $\begin{array}{l}\text { Days to } \\
\text { Harvest }\end{array}$ & MoAsects & $\begin{array}{l}\text { MOA } \\
\text { Code }^{1}\end{array}$ & Notes \\
\hline \hline $\begin{array}{l}\text { Xentari DF } \\
\text { (Bacillus thuringiensis } \\
\text { subspecies aizawai) }\end{array}$ & $0.5-2.0 \mathrm{lb}$ & 4 & 0 & caterpillars & $11 \mathrm{B1} 1$ & $\begin{array}{l}\text { Treat when larvae are young. } \\
\text { Thorough coverage is essential. } \\
\text { May be used in the greenhouse. } \\
\text { Can be used in organic produc- } \\
\text { tion. }\end{array}$ \\
\hline \hline
\end{tabular}

The pesticide information presented in this table was current with federal and state regulations at the time of revision. The user is responsible for determining the intended use is consistent with the label of the product being used. Use pesticides safely. Read and follow label instructions.

${ }^{1}$ Mode of Action codes for vegetable pest insecticides from the Insecticide Resistance Action Committee (IRAC) Mode of Action Classification v.3.3 October 2003. 1A. Acetylcholine esterase inhibitors, Carbamates 1B. Acetylcholine esterase inhibitors, Organophosphates

2A. GABA-gated chloride channel antagonists

$3 . \quad$ Sodium channel modulators

4A. Nicotinic Acetylcholine receptor agonists/antagonists, Neonicotinoids

5. Nicotinic Acetylcholine receptor agonists (not group 4)

6. Chloride channel activators

7A. Juvenile hormone mimics, Juvenile hormone analogues

7D. Juvenile hormone mimics, Pyriproxifen

9A. Compounds of unknown or non-specific mode of action (selective feeding blockers), Cryolite

9B. Compounds of unknown or non-specific mode of action (selective feeding blockers), Pymetrozine

11B1. Microbial disruptors of insect midgut membranes, B.t. var aizawai

11B2. Microbial disruptors of insect midgut membranes, B.t. var kurstaki

12B. Inhibitors of oxidative phosphorylation, disruptors of ATP formation, Organotin miticide

15. Inhibitors of chitin biosynthesis, type 0, Lepidopteran

16. Inhibitors of chitin biosynthesis, type 1 , Homopteran

17. Inhibitors of chitin biosynthesis, type 2, Dipteran

18. Ecdysone agonist/disruptor

20. Site II electron transport inhibitors

21. Site I electron transport inhibitors

22. Voltage-dependent sodium channel blocker

23. Inhibitors of lipid biosynthesis

25. Neuroactive (unknown mode of action)

26. Unknown mode of action, Azadirachtin

2 OMRI listed: Listed by the Organic Materials Review Institute for use in organic production.

${ }^{*}$ Restricted Use Only. 
Table 10. Breakeven production costs for sweet corn at various yield levels in the Miami-Dade County area, 2004-05.

\begin{tabular}{|c|c|c|c|c|c|c|}
\hline & \multirow[b]{2}{*}{ Cost per acre } & \multicolumn{5}{|c|}{ Yield (crates/acre) } \\
\hline & & 250 & 275 & 300 & 325 & 350 \\
\hline Variable costs & $\$ 1,857.05$ & $\$ 7.43$ & $\$ 6.75$ & $\$ 6.19$ & $\$ 5.71$ & $\$ 5.31$ \\
\hline Fixed costs & $\$ 1,514.75$ & $\$ 6.06$ & $\$ 5.51$ & $\$ 5.05$ & $\$ 4.66$ & $\$ 4.33$ \\
\hline Harvest cost/unit & & $\$ 2.98$ & $\$ 2.98$ & $\$ 2.98$ & $\$ 2.98$ & $\$ 2.98$ \\
\hline Total cost/unit & & $\$ 16.47$ & $\$ 15.24$ & $\$ 14.22$ & $\$ 13.35$ & $\$ 12.61$ \\
\hline
\end{tabular}

Table 11. Breakeven production costs for sweet corn at various yield levels in the Palm Beach County area, 2004-05.

\begin{tabular}{|lcrrrrr||}
\hline & \multicolumn{5}{c|}{ Yield (crates/acre) } \\
\cline { 2 - 6 } & Cost per acre & $\mathbf{2 0 0}$ & $\mathbf{2 2 5}$ & $\mathbf{2 5 0}$ & $\mathbf{2 7 5}$ & $\mathbf{3 0 0}$ \\
\hline \hline Variable costs & $\$ 1,638.22$ & $\$ 8.19$ & $\$ 7.28$ & $\$ 6.55$ & $\$ 5.96$ & $\$ 5.46$ \\
Fixed costs & $\$ 1,455.04$ & $\$ 7.28$ & $\$ 6.47$ & $\$ 5.82$ & $\$ 5.29$ & $\$ 4.85$ \\
Harvest cost/unit & & $\$ 3.28$ & $\$ 3.28$ & $\$ 3.28$ & $\$ 3.28$ & $\$ 3.28$ \\
Total cost/unit & & $\$ 18.75$ & $\$ 17.03$ & $\$ 15.65$ & $\$ 14.53$ & $\$ 13.59$ \\
\hline \hline
\end{tabular}

